\title{
Temperature drift compensation in Fourier-transform integrated micro-spectrometers
}

\section{Compensación de derivas térmicas en micro-espectrómetros integrados de trasformada de Fourier}

\author{
A. V. Velasco ${ }^{1,}{ }^{*}$, J. Galindo-Santos ${ }^{1}$, P. Cheben ${ }^{2}$, M. L. Calvo ${ }^{3, S}$, J. Schmid ${ }^{2}$, A. Delage ${ }^{2}$, Dan- \\ Xia Xu2, S. Janz ${ }^{2}$, P. Corredera ${ }^{1, S}$ \\ 1. Instituto de Óptica, Consejo Superior de Investigaciones Científicas, Madrid 28006, España \\ 2. National Research Council Canada, Ottawa, Ontario K1A 0R6, Canada. \\ 3. Facultad de Ciencias Físicas, Universidad Complutense de Madrid, Madrid 28040, España \\ ${ }^{(*)}$ E-mail: a.villafranca@csic.es \\ S: SEDOPTICA member \\ Received: 06/05/2015 Accepted: 01/12/2015 \\ DOI: $10.7149 /$ OPA.48.4.283
}

\begin{abstract}
:
In this work, we analyze the effect of temperature drifts in a spatial heterodyne Fourier-transform micro-spectrometer fabricated in a Silicon-on-insulator chip. The device comprises an array of 32 Mach-Zehnder interferometers implemented with microphotonic spirals of linearly increasing length. This configuration provides a resolution of $42 \mathrm{pm}$ in a footprint under $12 \mathrm{~mm}^{2}$. However, this resolution enhancement implies a greater sensitivity to environmental variations during the spectrometer operation. In this work, the effect of temperature drifts in the spectral retrieval algorithm is studied, and compensation techniques based on multiple calibration matrices is presented.
\end{abstract}

Key words: integrated optics, spectroscopy, Fourier transform, Silicon on insulator, temperature drift, spectral retrieval.

\section{RESUMEN:}

En este trabajo, analizamos el efecto de las variaciones de temperatura en un micro-espectrómetro de transformada de Fourier espacialmente heterodino integrado en un chip de silicio sobre aislante. El dispositivo comprende un array de 32 interferómetros Mach-Zehnder implementados con espirales microfotónicas de longitudes linealmente crecientes. Esta configuración permite alcanzar resoluciones de hasta $42 \mathrm{pm}$ en un dispositivo de tan sólo $12 \mathrm{~mm} 2$ de superficie. No obstante, el aumento de resolución implica una mayor sensibilidad del dispositivo a las condiciones ambientales de operación. En este trabajo se estudia el efecto de las derivas térmicas en el proceso de recuperación espectral del dispositivo y se presentan técnicas de compensación basadas en matrices de calibración múltiples.

Palabras clave: óptica integrada, espectroscopía, transformada de Fourier, silicio sobre aislante, deriva térmica, recuperación espectral.

\section{REFERENCES AND LINKS / REFERENCIAS Y ENLACES}

[1] E. D. Becker, T. C. Farrar, "Fourier transform spectroscopy," Science 178, 361-368 (1972). http://dx.doi.org/10.1126/science.178.4059.361

[2] P. R. Griffiths, J. A. De Haseth, Fourier Transform Infrared Spectrometry. New York, John Wiley \& Sons (2007).

http://dx.doi.org/10.1002/047010631X 
[3] J. M. Harlander, F. L. Roesler, J. G. Cardon, C. R. Englert, R. R. Conway, "A spatial heterodyne spectrometer for remote sensing of Earth middle aAtmosphere," Appl. Opt. 41, 1343-1345 (2002). http://dx.doi.org/10.1364/A0.41.001343

[4] M.-L. Junttila, J. Kauppinen, E. Ikonen, "Performance limits of stationary Fourier spectrometers," J. Opt. Soc. Am 8, 1457-1462 (1991). http://dx.doi.org/10.1364/JOSAA.8.001457

[5] M. Florjańczyk, C. Alonso-Ramos, P. Bock, A. Bogdanov, P. Cheben, Í. Molina-Fernández, S. Janz, B. Lamontagne, A. Ortega-Moñux, A. Scott, K. Sinclair, B. Solheim, D.-X. Xu, "Development of a Fouriertransform waveguide spectrometer for space applications," Opt. Quant. Elect. 44, 549-556 (2012). http://dx.doi.org/10.1007/s11082-012-9596-6

[6] P.J. Bock, P. Cheben, A.V. Velasco, J.H. Schmid, A. Delâge, M. Florjańczyk, J. Lapointe, D.-X. Xu, M. Vachon, S. Janz, M. L. Calvo, "Subwavelength grating Fourier-transform interferometer array in silicon-on-insulator," Laser Photonics Rev. 7, L67-L70 (2013). http://dx.doi.org/10.1002/lpor.201300063

[7] P. Jacquinot, "The luminosity of spectrometers with prisms, gratings, or Fabry-Perot etalons," J. Opt. Soc. Am. 44, 761-765 (1954). http://dx.doi.org/10.1364/JOSA.44.000761

[8] P. Cheben, "Wavelength dispersive planar waveguide devices: Echelle gratings and arrayed waveguide gratings", Chap. 5 in Optical Waveguides: From Theory to Applied Technologies. M. L. Calvo, V. Laksminarayanan, Edts, London, CRC Press, (2007).

[9] P. J. Bock, P. Cheben, J. H. Schmid, A. V. Velasco, A. Delâge, S. Janz, D.-X. Xu, J. Lapointe, T. J. Hall, M. L. Calvo, "Demonstration of a curved sidewall grating demultiplexer on silicon," Opt. Express 20, 19882-19892 (2012).

http://dx.doi.org/10.1364/OE.20.019882

[10] A. V. Velasco, P. Cheben, M. Florjańczyk, M. L. Calvo, "Spatial heterodyne Fourier-transform waveguide spectrometers," Progress in Optics 59, (2014). http://dx.doi.org/10.1016/b978-0-444-63379-8.00003-9

[11] J.-M. Lee, D.-J. Kim, H. Ahn, S.-H. Park, G. Kim, “Temperature Dependence of Silicon Nanophotonic Ring Resonator With a Polymeric Overlayer," J. Lightwave Technol. 25, 2236 (2007). http://dx.doi.org/10.1109/JLT.2007.899792

[12] J. H. Schmid, M. Ibrahim, P. Cheben, J. Lapointe, S. Janz, P. J. Bock, A. Densmore, B. Lamontagne, R. Ma, W. N. Ye, D.-X. Xu, “Temperature-independent silicon wavelength grating waveguides," Opt. Lett. 36, 2110-2112 (2011). http://dx.doi.org/10.1364/OL.36.002110

[13] A. V. Velasco, P. Cheben, P. J. Bock, A. Delage, J. H. Schmid, J. Lapointe, S. Janz, M. L. Calvo, D.-X. Xu, M. Florjańczyk, M. Vachon, "High-resolution Fourier-transform spectrometer chip with microphotonic silicon spiral waveguides," Opt. Lett. 38, 706-708 (2013). http://dx.doi.org/10.1364/OL.38.000706

[14] P. Cheben, P. J. Bock, J. H. Schmid, J. Lapointe, S. Janz, D.-X. Xu, A. Densmore, A. Delâge, B. Lamontagne, T. J. Hall, "Refractive index engineering with subwavelength gratings for efficient microphotonic couplers and planar waveguide multiplexers," Opt. Lett. 35, 2526-2528 (2010). http://dx.doi.org/10.1364/OL.35.002526

[15] K. Okamoto, H. Aoyagi, K. Takada, "Fabrication of Fourier-transform, integrated-optic spatial heterodyne spectrometer on silica-based planar waveguide," Opt. Lett. 35, 2103-2105 (2010). http://dx.doi.org/10.1364/OL.35.002103

[16] G. H. Golub, C. Reinsch, "Singular value decomposition and least squares solutions," Numerische Mathematik 14, 403 (1970).

http://dx.doi.org/10.1007/BF02163027

\section{Introducción}

La espectroscopía de Transformada de Fourier (FT) es una poderosa herramienta de análisis espectral basada en la realización de medidas interferométricas con diferencias de caminos ópticos variables [1, 2], tradicionalmente realizadas mediante un espejo móvil. Los espectrómetros FT estacionarios, también conocidos como espectrómetros de transformada de Fourier espacialmente heterodinos (SHFT), evitan el uso de elementos mecánicos móviles realizando múltiples medidas interferométricas en paralelo con 
diferencias de caminos ópticos fijas [3, 4]. Se genera así un patrón espacial de salida estacionario que contiene la información equivalente al barrido de la línea de retardo óptico. Puesto que la configuración SHFT únicamente requiere elementos pasivos, es posible su implementación en óptica integrada mediante guías de ondas planas $[5,6]$. Los espectrómetros SHFT integrados pueden ser diseñados con una elevada apertura de entrada, proporcionando una notable ventaja en términos de rendimiento óptico (étendue) [7] respecto a otros espectrómetros integrados convencionales basados en AWGs [8] o redes de difracción [9], limitados a una única guía de onda monomodo de entrada. Adicionalmente, su implementación en silicio sobre aislante (SOI) permite integrar interferómetros de gran longitud en una superficie muy reducida, aumentando así la resolución del dispositivo [10].

Para el correcto análisis de los patrones estacionarios proporcionados por los espectrómetros SHFT, resulta imprescindible un conocimiento preciso de las diferencias de caminos ópticos implementadas por el dispositivo. No obstante, las variaciones en las condiciones ambientales durante la operación del espectrómetro pueden modificar significativamente el índice de refracción efectivo de las guías de onda $[11,12]$, alterando las diferencias de caminos ópticos y el interferograma resultante. En el presente trabajo, presentamos un estudio sobre los efectos de las variaciones de temperatura en la recuperación espectral realizada por un micro-espectrómetro SHFT integrado en SOI, y presentamos alternativas para su compensación.

\section{Micro-espectrómetro de Transformada de Fourier}

\section{2.a. Principio de operación}

El espectrómetro SHFT comprende un array de $N$ interferómetros Mach-Zehnder (MZI) con diferencias de caminos ópticos linealmente crecientes entre los brazos de cada interferómetro. Dentro del rango espectral libre (FSR) del dispositivo, la relación entre el interferograma de salida $I\left(x_{i}\right)$ y la señal de entrada queda determinada por:

$$
I\left(x_{i}\right)=\int_{0}^{F S R} B(\bar{\sigma}) \cos \left(2 \pi \bar{\sigma} x_{i}\right) d \bar{\sigma}
$$

donde $B$ es la densidad espectral de entrada, $x_{i}$ es el retardo óptico asociado al $i$-ésimo MZI, y $\bar{\sigma}=\sigma-\sigma_{L}$ es el número de onda relativo al número de onda de Littrow [10]. Esta relación es unívoca para dispositivos ideales sin errores de fase, permitiendo recuperar el espectro de la señal de entrada mediante técnicas numéricas basadas en transformada discreta de Fourier.

La resolución $(\delta \lambda)$ y el rango espectral libre del espectrómetro quedan determinados por el número de interferómetros y la máxima diferencia de camino ópticos del array $\left(\Delta L_{\max }\right)$ :

$$
\begin{gathered}
\delta \lambda=\frac{\lambda_{0}^{2}}{\Delta L_{\max } n_{g}} \\
\mathrm{FSR}=\delta \lambda \frac{N}{2}
\end{gathered}
$$

donde $\lambda_{0}$ es la longitud de onda central de operación y $n_{g}$ es el índice de grupo de las guías de onda.

\section{2.b. Diseño y fabricación}

El espectrómetro SHFT particular bajo análisis comprende 32 MZI implementados mediante guías de onda de silicio con una anchura de $450 \mathrm{~nm}$. Cada interferómetro está formado por un brazo recto de longitud fija y una espiral microfotónica de longitud linealmente creciente [13]. El alto contrasto de índice y el consiguiente confinamiento de modo de la plataforma SOI permite implementar espirales extremadamente compactas, alcanzando una longitud de $\Delta L_{\max }=1.13 \mathrm{~cm}$ en un diámetro de tan sólo 270 $\mu \mathrm{m}$. Para $\lambda_{0}=1550 \mathrm{~nm}$ y $n_{g}=4.38$, estas dimensiones permiten obtener una resolución de 42 pm y un FSR de $0.75 \mathrm{~nm}$ en una superficie total menor de $12 \mathrm{~mm}^{2}$. El índice de grupo se ha estimado mediante simulación de las guías de onda por software de diferencias finitas en el dominio del tiempo, siendo validado experimentalmente a través del periodo de la transmitancia de los MZI. El dispositivo comprende acopladores basados de redes sub-wavelength [14] en las caras de entrada y salida del chip para optimizar el acoplo y reducir los efectos Fabry-Perot ocasionados por reflexiones. 
El espectrómetro se fabricó en substratos SOI con una capa de silicio de $0.26 \mu \mathrm{m}$ de espesor sobre una capa de dióxido de silicio de $2 \mu \mathrm{m}$ de espesor. Los patrones de todas las estructuras del microespectrómetro fueron definidas en un único paso mediante litografía de haz de electrones, utilizando una resistencia de silsequioxano de hidrógeno (HSQ) de alto contraste. La transferencia de los patrones se realizó mediante grabado de iones reactivos por plasma acoplado inductivamente (ICP-RIE). Los circuitos fotónicos resultantes se recubrieron mediante polímero SU-8. Las estructuras espirales de los interferómetros fabricados pueden observarse en la figura 1.
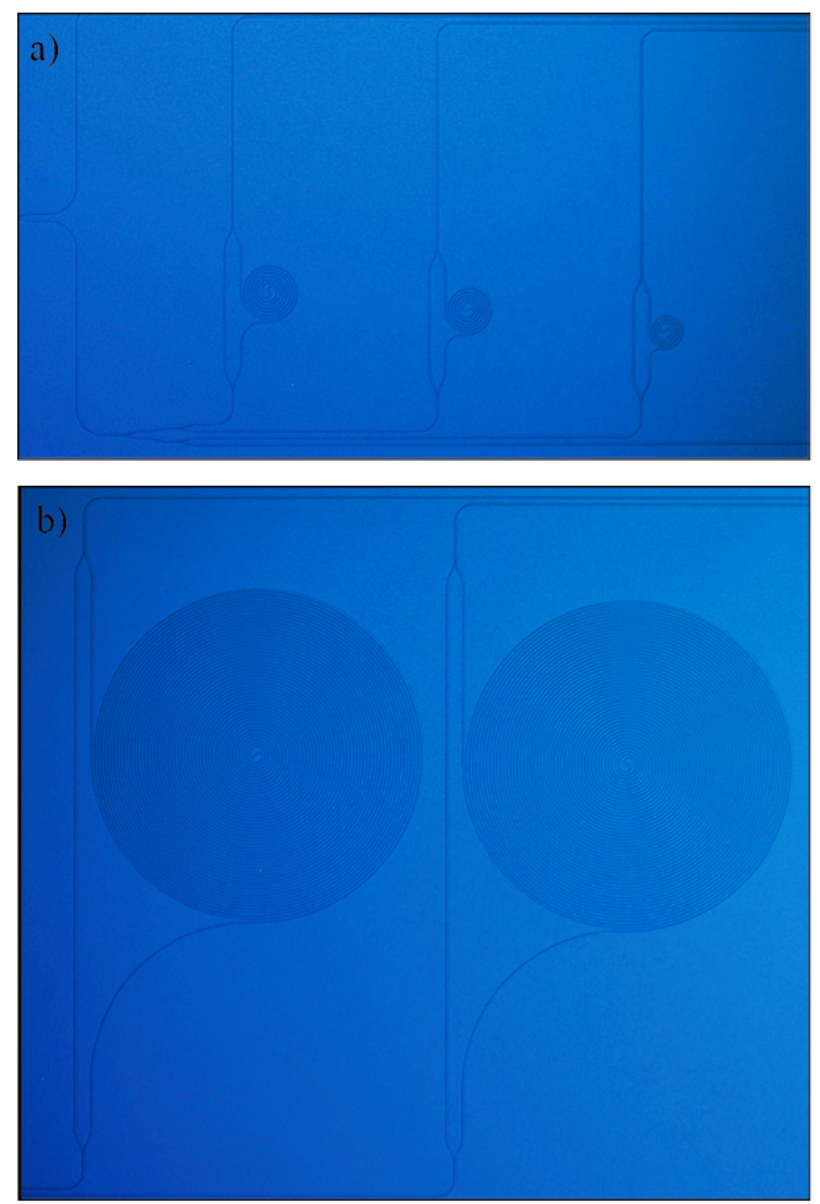

Fig.1. Imágenes de microscopía SEM de las estructuras espirales de menor (a) y mayor (b) longitud del espectrómetro fabricado.

\section{2.c. Recuperación espectral}

Para analizar correctamente el interferograma proporcionado por un espectrómetro SHFT, es necesario considerar dos desviaciones inherentes a los dispositivos fabricados respecto al modelo teórico proporcionado por Eq. (1). En primer lugar, las perdidas de propagación reducen la visibilidad de la señal de salida en función de la longitud de la espiral microfotónica. En segundo lugar, pequeñas imperfecciones de fabricación dan lugar a errores de fase en las señales sinusoidales generadas por el array de MZI, impidiendo que se verifique la condición de alineación de la longitud de onda de Littrow. Los cambios en la visibilidad a lo largo del array son fácilmente compensables mediante técnicas de normalización, pero los errores de fase afectan directamente a la ortogonalidad de la base de la transformación realizada por el dispositivo.

Los errores de fase pueden compensarse activamente mediante circuitos térmicos dedicados [15], pero suponen un aumento notable del tamaño del dispositivo y de la complejidad de la medida. Alternativamente, es posible utilizar algoritmos de recuperación espectral que incorporan técnicas numéricas de compensación basadas en matrices de calibración, tal y como se detalla en [10]. Se incorporan así al algoritmo las desviaciones de fase y amplitud particulares de cada dispositivo fabricado. Un ejemplo experimental de matriz de calibración para polarización TM y $\lambda 0=1.55 \mu \mathrm{m}$ se presenta en la figura 2 . 


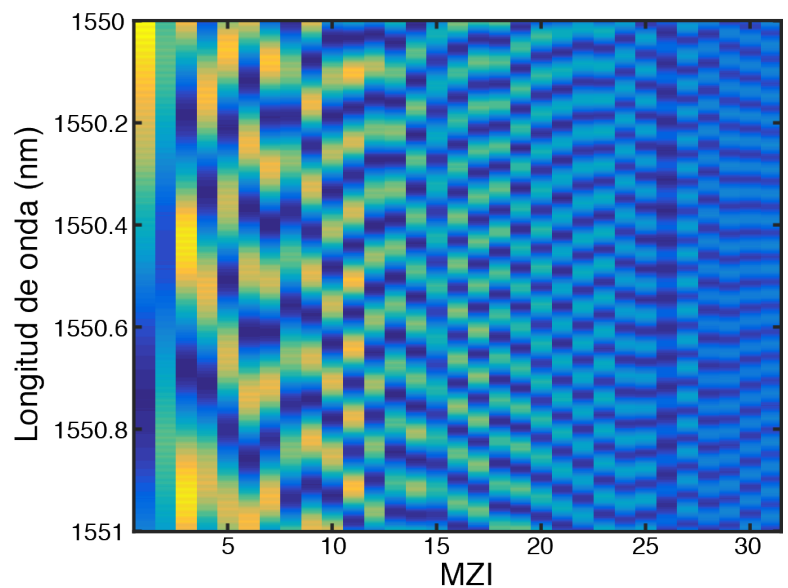

Fig. 2: Mapa de calibración para polarización TM del microespectrómetro formado por las distribuciones de potencia de salida de los 32 interferómetros Mach-Zehnder en un rango de $1 \mathrm{~nm}$ iniciado en $1.55 \mu \mathrm{m}$.

En el dispositivo particular bajo análisis, se midieron pérdidas de propagación de $-4 \mathrm{~dB} / \mathrm{cm}$ y pérdidas por curvatura de $-1.7 \mathrm{~dB} / \mathrm{cm}$ en las estructuras espirales. Asimismo, se midió un índice de grupo promedio de 4.38, con desviaciones menores del $1 \%$ en los incrementos lineales de las diferencias de caminos ópticos a lo largo del array.

\section{Efectos térmicos}

\section{3.a. Alteración del interferograma}

A una longitud de onda de $1.55 \mu \mathrm{m}$, las guías de onda utilizadas presentan un coeficiente termo-óptico $d n_{\text {eff }} / d T$ de $1.8 \times 10^{-4} \mathrm{~K}^{-1}$ para polarización TE y de $1.2 \times 10^{-4} \mathrm{~K}^{-1}$ para polarización TM [12], donde $n_{\text {eff }}$ es el índice de refracción efectivo de la guía de onda y $T$ es su temperatura. Un cambio de temperatura del espectrómetro durante su operación provoca por lo tanto un cambio en el periodo de la función de transferencia de cada MZI dependiente de su diferencia de camino óptico, con la consiguiente alteración del interferograma generado. Cuanto mayor es $\Delta L_{\max }$, y por lo tanto la resolución del espectrómetro, más sensible resulta el dispositivo a los cambios en la temperatura.

La figura 3 muestra la variación térmica que genera un desplazamiento de una franja completa en la función de transferencia del interferómetro con mayor diferencia de caminos ópticos, en función de la resolución que se obtiene con dicha $\Delta L_{\max }$, para la geometría de guía de onda y para la longitud de onda central bajo análisis. En particular, para el dispositivo fabricado, una variación de $1.2{ }^{\circ} \mathrm{C}$ genera un desplazamiento de una franja completa para polarización TM, viéndose reducida dicha variación a $0.8{ }^{\circ} \mathrm{C}$ en el caso de polarización TE.

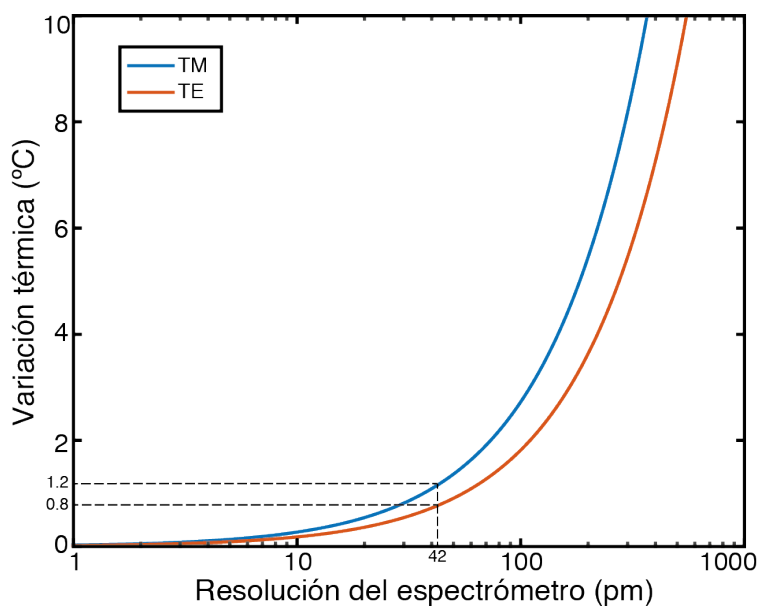

Fig. 3: Variación de temperatura asociada a un desplazamiento de una franja completa del interferómetro de mayor longitud, en función de la resolución del espectrómetro, para polarización TE (rojo) y TM (azul) a 1,55 $\mu$ m. Se ha destacado el punto de la curva correspondiente al dispositivo bajo análisis. 
Puesto que el algoritmo de recuperación espectral se basa en matrices de calibración que definen la función de transferencia de cada MZI, es necesario que dichas funciones de transferencia permanezcan constantes entre la calibración y la operación del dispositivo. En caso de producirse cambios de temperatura entre ambos instantes, la inadecuación de la matriz de calibración genera errores en la recuperación espectral, tal y como se observa en la figura 4. Las medidas para la calibración y el análisis del dispositivo se realizaron con un laser de semiconductor sintonizable con una resolución de $0.1 \mathrm{pm}$. Se incluyó una etapa Peltier para estabilización térmica y controladores de polarización a la entrada del dispositivo debido a la elevada birrefringencia propia de la plataforma SOI, siendo seleccionada en este caso la polarización TM. El interferograma formado por las salidas de los interferómetros fue capturado mediante un disparo único de una cámara CCD infrarroja de alta sensibilidad.

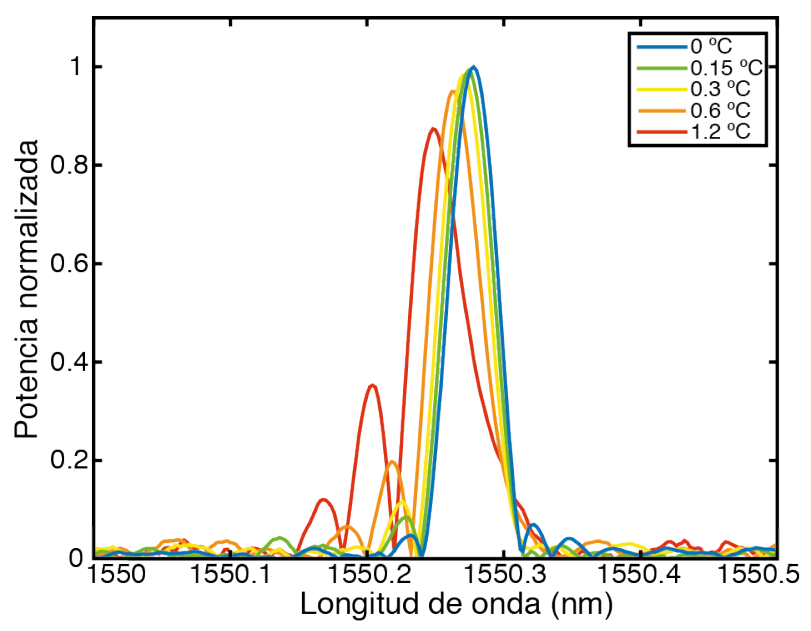

Fig. 4: Recuperación espectral de una señal monocromática en función de la diferencia de temperatura entre la medida del interferograma y el mapa de calibración correspondiente.

Debido a que la variación de temperatura afecta a cada MZI en diferente grado según su diferencia de caminos ópticos, se aprecian en los espectros recuperados de la señal monocromática tanto un desplazamiento de la longitud de onda central, como una deformación progresiva del espectro, dando lugar a la aparición de artefactos en los laterales del lóbulo principal.

\section{3.b. Técnicas de compensación}

Para mitigar los efectos de las derivas térmicas y reducir las restricciones impuestas sobre el sistema de estabilización de temperatura, hemos adaptado el algoritmo de recuperación espectral descrito en [10]. En lugar de utilizar una única matriz de calibración $C$, se miden múltiples matrices de calibración $C_{j}$ a múltiples temperaturas equiespaciadas $T_{j}$. Cada matriz contiene los interferogramas de salida generados por una señal monocromática sintonizable que muestrea el rango espectral libre del dispositivo a las longitudes de onda $\lambda_{k}$, de modo que:

$$
I\left(x_{i}, T_{j}, \lambda_{k}\right)=B\left(\lambda_{k}\right) \times C_{j}
$$

Una vez realizada la calibración sensible a temperatura, la matriz $C_{j}$ a utilizar en cada recuperación espectral se selecciona a través de una medida auxiliar previa o simultánea a la operación del dispositivo. Dicha medida auxiliar puede implementarse bien mediante una medición directa de alta precisión de la temperatura del chip fotónico $\left(T_{a u x}\right)$, bien mediante la captura del interferograma $I_{a u x}$ asociado a una señal de referencia de espectro conocido $B_{\text {ref. }}$ En el primer caso, se elige directamente la matriz $C_{j}$ cuya temperatura $T_{j}$ es más próxima a $T_{a u x}$. En el segundo caso, se selecciona la matriz $C_{j}$ que minimiza la expresión:

$$
\sum_{i=1}^{N}\left|I_{\text {aux }}-B_{\text {ref }} \times C_{j}\right|
$$

Una vez seleccionada la matriz $C_{j}$ adecuada, el espectro de la señal a caracterizar se obtiene multiplicando su interferograma por $C_{j}^{+}$, matriz pseudoinversa de $C_{j}$ calculada mediante descomposición en valores singulares [16]. La medida del interferograma completo en un sólo disparo permitida por la configuración SHFT permite minimizar el tiempo de medida de la señal de referencia, garantizado así la correcta recuperación del espectro de la señal a caracterizar. Al igual que en el caso del algoritmo de recuperación 
espectral utilizado en [10], el uso de matrices experimentales de calibración garantiza la compensación inherente de desviaciones de fase y de variaciones de contraste a lo largo del array de MZI. La figura 5 muestra un ejemplo particular de aplicación de la técnica de compensación de efectos térmicos descrita.

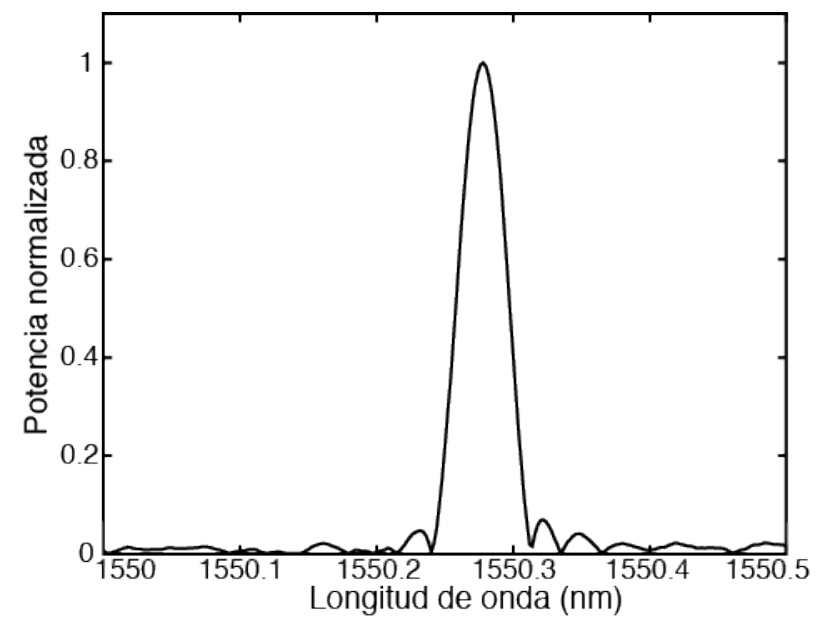

Fig. 5: Recuperación espectral de una señal monocromática con deriva térmica compensada mediante matrices de calibración múltiples.

\section{Conclusiones}

Se han presentado los efectos ocasionados por variaciones térmicas en espectrómetros de transformada de Fourier espacialmente heterodinos integrados en guías de onda con base de silicio. Las diferencias de temperatura entre la calibración y la operación del dispositivo introducen desplazamientos frecuenciales y artefactos en el algoritmo de recuperación espectral. Los requisitos de estabilización térmica impuestos por estos efectos resultan más restrictivos según aumenta la resolución del espectrómetro. Para mitigar dichos requisitos, se han presentado técnicas de compensación basadas en matrices múltiples de calibración integradas en el algoritmo de recuperación espectral. Unidas a la elevada resolución y el pequeño tamaño del dispositivo, las técnicas presentadas permiten aplicar la tecnología SHFT a sensores médicos y ambientales desechables, instrumentación de espectroscopía y de mano y sensado para plataformas de microsatélites.

\section{Agradecimientos}

Este trabajo ha sido apoyado económicamente por el Ministerio de Economía de España a través de los proyectos TEC2012-37958-C02-02 y TEC2012-37958-C02-01, la Comunidad de Madrid a través de S2013/MIT-2790, y EURAMET a través del proyecto JRP-i22 14IND13 Photind. Agradecemos a Martin Vachon la ayuda proporcionada durante la caracterización del dispositivo. 